\title{
Insights Into Amino Acid Metabolism and Incorporation by Compound- Specific Carbon Isotope Analysis of three-Spined Sticklebacks
}

Maik Jochmann ( $\triangle$ maik.jochmann@uni-due.de)

University of Duisburg-Essen

\section{Tobias Hesse}

University of Duisburg-Essen

Milen Nachev

University of Duisburg-Essen

Shaista Khaliq

University of Duisburg-Essen

Frederik Franke

Bayerische Landesanstalt für Wald und Forstwirtschaft

Jörn Scharsack

Johann Heinrich von Thünen-Institut

Joachim Kurtz

University of Münster

\section{Bernd Sures}

University of Duisburg-Essen

Torsten Schmidt

University of Duisburg-Essen

\section{Research Article}

Keywords: amino acids, compound-specific stable isotope analysis, feeding experiment, metabolic pathways, stickleback

Posted Date: December 20th, 2021

DOI: https://doi.org/10.21203/rs.3.rs-1156412/v1

License: (c) (1) This work is licensed under a Creative Commons Attribution 4.0 International License.

Read Full License 


\section{Abstract}

Interpretation of isotope data is of upmost importance in ecology to build sound models for the study of animal diets, migration patterns and physiology. However, our understanding of isotope fractionation and incorporation into different consumer tissues is still limited. We therefore measured the $\delta^{13} \mathrm{C}$ values of individual amino acids from muscle and liver tissue of three-spined sticklebacks (Gasterosteus aculeatus) over time in a controlled feeding experiment. The isotope signatures of amino acids in the liver quickly responded to even small shifts in dietary isotope compositions, whereas muscle tissue remained isotopically constant over time. No significant fractionation between diet and fish tissues was observed for half of the amino acids in both liver and muscle tissue, supporting the idea of direct nutrient routing on a protein rich diet. Small isotopic differences were observed for alanine, glycine and serine between diet and liver, indicating that metabolic processes such as glycolysis or gluconeogenesis can be tracked by the isotope signatures of their main substitutes. We demonstrate that compound-specific isotope analysis has great potential to investigate the main metabolic pathways of organisms and suggest further investigations using isotopically enriched materials to facilitate the correct interpretation of ecological field data.

\section{Introduction}

Stable isotope analysis (SIA) of carbon is a powerful tool in ecological studies to investigate resource utilization, foraging behavior and migration patterns of animals ${ }^{1-3}$. The underlying principle is that the carbon isotope composition of diets is mostly retained by consumers, with only little fractionation occurring during incorporation. In bulk stable isotope analysis (BSIA), this fractionation increases the carbon isotope signature of consumers by $0-1 \%{ }^{4,5}$ and the carbon isotope signature of an individual is therefore mostly depending on the isotope composition of primary producers. This enables tracking of nutrients from the base of food webs to higher trophic level predators. However, there can be deviations from this pattern depending on species, analyzed tissue and diet composition ${ }^{6,7}$. A more recent approach, compound specific isotope analysis (CSIA), was enabled by analyzing individual compounds, rather than bulk tissues. Where BSIA of carbon typically shows little fractionation between diet and consumer, individual constituents such as amino acids (AA) can have higher fractionation, depending on which compounds they are routed from ${ }^{3,8-12}$.

AAs can be divided into essential amino acids (EAA) and non-essential amino acids (NEAA). EAAs cannot be synthesized by higher organisms and therefore need to be taken up directly from dietary sources, leading to no or very little isotope fractionation because these nutrients are traversing the food chain mostly unchanged from primary producers to top predators. This approach is called isotope fingerprinting and has been used for ecological studies to investigate the carbon flow and nutrient allocation of end-consumers by comparing the isotope signature of selected EAAs to those of primary producers, which should be roughly identical if the food chains are connected ${ }^{3,13-15}$. NEAAs on the other hand can be synthesized de novo in higher organisms and therefore originate either from dietary sources 
and/or other metabolic compounds during metabolism and conversion of nutrients, such as lipids and carbohydrates. The classification of AAs into essential and non-essential, however, is not always easy, as the requirement of some NEAAs can sometimes outmatch the organism's ability to synthesize these compounds, rendering them temporarily or conditionally essential ${ }^{16}$, with Glu and Pro being two examples. Another example would be tyrosine (Tyr), which can be synthesized in higher organisms but is derived from phenylalanine (Phe), which is an EAA. The carbon isotope signature of Tyr therefore typically falls into the same range as $\mathrm{Phe}^{12}$, although technically it should be considered non-essential.

Most studies on stable isotopes typically only consider whole body or muscle tissue ${ }^{17}$, as these are the easiest to access. The isotope turnover and incorporation rate can, however, be very low and as a result, turnover in muscle tissue is more affected by consumer physiology and growth phases during which protein synthesis and deposition occurs. The liver responds much more quickly to dietary isotopic changes, as its regulatory activities require continuous protein turnover and have shown to be more responsive to short-term changes in isotope signature ${ }^{18-22}$. In addition, the liver plays an important role in the metabolism of AAs and metabolic processes such as glycolysis and gluconeogenesis for energy production and storage ${ }^{16,23,24}$, which makes the liver an ideal tissue for studying these processes. This is especially true for CSIA, since the key compounds participating in glycolysis and gluconeogenesis are AAs, carbohydrates and lipids ${ }^{25}$. Regarding energy metabolism, AAs can then be further divided into glycogenic and ketogenic categories, depending on the pathways by which they are metabolized. Glycogenic AAs are converted mainly to glucose via pyruvate, whereas ketogenic AAs are converted to ketone bodies. The differentiation is, however, not so easy, as some AAs can be metabolized via both pathways. The most important AAs for gluconeogenesis in humans are Ala and Glu ${ }^{26,27}$, with Ala serving as a "shuttle" to transport three-carbon skeletons from muscle to liver during proteolysis. Other important AAs participating in gluconeogenesis are Gly, Ser and Asp. Whether these compounds are directly routed from diet or synthesized de novo is further depending on dietary composition, whereas consumers fed on diets with high protein contents tend to directly route also NEAAs in order to preserve energy $11,13,28,29$. Studying the isotope fractionation of metabolic pathways involved in the synthesis and catabolism of NEAA and other macronutrients helps researchers to interpret the ability of omnivorous and herbivorous animals to maintain energy and nutrient homeostasis on diets with different protein, lipid and carbohydrate compositions.

Since most studies using SIA are field based, there was an initial call for more laboratory SIA studies in $1997^{30}$ to better understand the fundamental principles of isotopic incorporation, trophic discrimination and isotope routing. Although the number of laboratory-based studies has increased since then, the call was renewed in $2009^{31}$, to facilitate correct interpretation of field studies. Few controlled feeding experiments so far examined the carbon isotope fractionation of individual AAs between diet and fish consumers, with varying magnitudes and directions of carbon isotope fractionation reported $10-13,29$. McMahon et al. (2010), for example, found ${ }^{13} \mathrm{C}$-depleted isotope signatures of Gly between Common Mummichogs (Fundulus heteroclitus) and one of their diets, whereas Rogers et al. (2019) found 
significantly ${ }^{13} \mathrm{C}$-enriched Gly carbon isotope signatures of Chinook Salmon (Oncorhynchus tshawytscha) reared on the same diet. This example demonstrates that clear differences in individual AA isotope fractionations occur among fish species.

The three-spined stickleback (Gasterosteus aculeatus) is a well-studied model fish in ecology,

evolutionary biology and parasitology ${ }^{32,33}$, yet no carbon isotope analysis of amino acids of sticklebacks has been done to this date. There are some studies investigating carbon and nitrogen BSIA of threespined sticklebacks ${ }^{34-37}$, but those were also field-based, investigating differences associated with sex, armor, phenotypes, genetics and host-parasite relationships. We conducted a laboratory-based feeding experiment, where three-spined sticklebacks were reared on a protein rich diet over the course of four months. To contribute to the advancement of our understanding of isotope incorporation, discrimination and routing, we measured the carbon isotope signature of individual AAs from muscle, liver and dietary samples by Liquid Chromatography Isotope Ratio Mass Spectrometry (LC-IRMS). Previous BSIA of dietary samples (data not published) revealed a minor carbon isotope shift over the course of the experiment, which is likely to be transferred to fish tissue to varying degrees, depending on tissue type. This is the first time that carbon isotope signatures of AAs were measured for three-spined sticklebacks on a time series in a controlled feeding experiment. Data was analyzed to specifically test and answer the following three main hypotheses:

1) NEAAs in stickleback tissue will be preferentially routed to diet in the same manner as EAAs, since fish are fed on high protein contents

2) Liver AA carbon isotope signatures change over the course of experiment and follow the isotope shift of dietary samples, whereas AA isotope signatures in muscle tissue remain constant or shift only slightly over time.

3) Glycogenic AAs in the liver will show different carbon isotope patterns compared to muscle and diets based on their metabolic role, but we can't predict in what way.

\section{Results}

\section{Isotope shift over time}

One-way ANOVA for each AA with time points (days) as independent variable and $\delta^{13} \mathrm{C}$ values as dependent variable of all dietary samples revealed a significant isotope shift between time points for all AAs except His ( $D F=3,8, p<0.01$, Table S2, Supporting Information). Over time, average isotope signatures of AAs in dietary samples changed by -1.6 to $-1.0 \%$ o between 30 and 60 days, followed by another change of -2.6 to $-0.9 \%$ o between 60 and 90 days and a final change of 0 to $+1.5 \%$ o between 90 and 120 days. A general trend can be seen where the isotope signature of each dietary AA decreased between 30 and 60 days as well as 60 and 90 days, followed by an increase in isotope composition 
between 90 and 120 days. The biggest differences between isotope signatures ( -2 to $-4 \%$ ) were therefore observed between 30 and 90 days, marking the time points where diets had the highest and lowest $\delta^{13} \mathrm{C}$ values (Figure 1 and Figure 2). Isotope signatures of AAs in the liver were significantly different over time for Ala $\left(F_{3,8}=6.8, p=0.004\right)$, Asp $\left(F_{3,8}=9.2, p=0.001\right)$, Arg $\left(F_{3,8}=13.7, p<0.001\right)$, Lys $\left(F_{3,8}=18.4, p<0.001\right)$, Phe $\left(F_{3,8}=8.9, p=0.001\right)$ and $\operatorname{Tyr}\left(F_{3,8}=20.8, p<0.001\right)$. Gly, Ser, Glu, Pro and Thr were not significantly different between time points in the liver, although Glu was just slightly above the significance level $\left(F_{3,8}=5.1, p=0.011\right)$. Although not all AAs in the liver revealed a significant isotope shift, the general trend of decreasing $\delta^{13} \mathrm{C}$ values from 30 to 60 days and from 60 to 90 days followed by an increase from 90 to 120 days was still visible, comparable to the shift in dietary samples. In contrast to liver samples, no AA in muscle tissue showed any significant differences between time points.

\section{Isotope fractionation between tissues}

Muscle and liver tissue of sticklebacks were separated by ASCA on the first principal component of factor 1 (sample type, Figure 3), which contributed $22.4 \%$ to the total variance. NEAAs were the main driver of separation on the first principal component (81.8\% variance) with loadings above 0.3 for Ala, Asp, Glu and Ser, whereas Gly was the only NEAA with a negative loading of under -0.2. Ser had the overall highest loading of 0.7 on PC1. All EAAs and Pro had loadings of under \pm 0.2 and therefore little to no effect on PC1 separation. Individual scorings of liver samples were negative on PC1, whereas muscle tissue had almost exclusively positive scorings. Scorings of dietary samples gathered around $\sim 0$. PC2 showed no clear separation between tissue types and was mostly loaded with EAAs, except for Arg and Lys. Tyr, Phe and Pro had the highest loadings of over 0.2 , whereas Thr showed a noticeable negative loading of under -0.3. Scores of muscle and liver samples on PC2 showed no clear trend with a slight tendency to be more negative. Dietary samples showed positive scorings on PC2, which led to an almost distinct cluster of dietary samples with a slight overlap in the elliptical confidence ring with liver samples. Arg and Lys were the only amino acids which had almost no impact on separation between tissues for both PCs. Factor 2 (days) of the ANOVA simultaneous component analysis had an effect of $35.5 \%$ on system variance but will not be discussed because at this stage it is of no further interest for data interpretation.

Differences of individual AAs between sample types were tested by one-way ANOVA for each AA and sampling days, with tissue type as independent variable and $\delta^{13} \mathrm{C}$ in $\%$ os dependent variable, followed up by Tukey post-hoc tests and a significance level a of 0.01 (Table S3 and S4, Supporting Information). All NEAAs except Pro showed significant differences between tissue types at least once throughout the days ( $D F=2,10, p<0.01$,), although differences of Asp and Glu were only seen between liver and muscle tissue and not compared to dietary tissue. Post hoc comparisons of mean values by Tukey tests showed that diet and liver tissue were isotopically different for Ala after 60 days $(p=0.007)$, Gly at 90 days $(p=$ $0.008)$ and Ser at 30 days $(p=0.01)$, whereas diet and muscle tissue only showed significant differences for Ala at 90 days $(p=0.001)$. Isotopic differences between muscle and liver tissue were more abundant, especially for Ala, Asp and Ser (Table S3, Supporting Information). The liver showed a general trend of being isotopically ${ }^{13} \mathrm{C}$-depleted compared to diet and muscle tissue, except for Gly, where the liver had ${ }^{13} \mathrm{C}$-enriched isotope signatures at 90 and 120 days. Differences between tissue types for EAAs were only 
significant for Phe and Tyr (DF $=2,8 ; p<0.01)$. These differences occurred in both cases mainly between fish tissue (either muscle or liver) compared to diets and not between liver and muscle themselves. Muscle and liver tissue were generally ${ }^{13} \mathrm{C}$-depleted compared to diets by around $-1 \%$. Arg, Lys and Thr showed no significant isotopic differences between tissue types.

\section{Isotope fractionation of Histidine}

The most significant isotope fractionation was observed for His between diet and both liver and muscle. His in liver was isotopically enriched with mean differences of $+8.2 \%$ o compared to dietary samples (DF $=$ $2,8 ; p<0.001)$. Fractionation of His between fish muscle and dietary samples was lower compared to liver tissue, but still significant at each day with $p$-values $<0.001$ and mean differences of $+5.0 \%$. Differences of His isotope signatures between muscle and liver tissue were also significant $(p<0.001)$ except at 90 days and mean differences of $-3.2 \%$. His isotope signatures in tissues were not significantly different over time, although dietary samples had a p-value of 0.016 and were therefore close to 0.01 , with the same isotopic trend of decreasing signatures at 60 and 90 days, followed by an enrichment of isotope signatures at 120 days.

\section{Discussion}

\section{Isotope signature over time}

The initial amino acid isotope signature of mosquito larvae used for feeding shows typical patterns also seen in other studies, where Gly and Ser are the most ${ }^{13} \mathrm{C}$-enriched amino acids and EAAs are isotopically

${ }^{13} \mathrm{C}_{\text {-depleted }}{ }^{3,8-13,15,29,38,39}$. The cause for the isotope shift of dietary AAs over time in our study remains unknown, but the fact that all AAs have a similar shift indicates that different source or batch material of mosquito larvae might have been used and mixed throughout the experiment. The response of sticklebacks to the introduced dietary isotope shift was different between muscle and liver tissue. The carbon isotope signature of AAs in muscle samples shifts less over the sampling period, shown by oneway ANOVA revealing no significant difference of any AA between sampling days. This means in our case, muscle tissue of sticklebacks did not significantly reflect changes in dietary isotope compositions of $\pm 2 \%$ on 30 day intervals. Although small trends in the average isotope values were visible in muscle over time, those changes were statistically not significant and overshadowed by natural variations of isotope signatures between individuals. This supports the idea that AAs from muscle tissue are a more conservative indicator of long-term dietary intake in fish, similar to what was previously described for BSIA ${ }^{20,22}$.

Liver tissue, on the other hand, showed significant isotopic differences over time, indicating that the high nutrient turnover rate of the liver is reflected in its carbon isotope signature of AAs and even short-term changes in dietary isotope compositions are visible. EAAs showed the highest differences (one-way ANOVA, $p \leq 0.001$ ) and might be indicative of high turnover for these compounds because they must be rooted from dietary sources. Only Ala and Asp showed significant differences over time from NEAAs in 
liver. Both AAs are important energy substrates in fish and precursors for gluconeogenesis ${ }^{40}$, which is the metabolic pathway in the liver to synthesize glucose from other carbon substrates. Fish therefore have a high Ala and Asp demand in liver tissue, which might outmatch the fish's ability to sufficiently synthesize these compounds and lead to an increased incorporation or turnover from dietary sources. According to our results, liver carbon isotope signatures of Ala, Asp, Arg and Lys might be useful proxies to track consumer diets of small teleost fish on protein rich diets and on time scales of at least 30-day intervals. This information could possibly be expanded to non-protein rich diets for Arg and Lys, due to the necessity of eukaryotes to directly incorporate these nutrients from dietary source, regardless of their composition ${ }^{13}$. Although we did not quantify the exact cellular turnover rate of individual AAs in stickleback tissues, knowing an estimated timeline for evaluating the diet of an animal is useful for ecological studies investigating e.g. migration or seasonal changes of food sources. It has been shown that choosing different tissue types in individuals enables researchers to explore temporal and spatial resource use of animals and it is critical to consider incorporation rates of isotope signatures into these tissues ${ }^{41,42}$. However, most studies so far on isotopic incorporation and turnover rates are still based on BSIA ${ }^{43-45}$. Enhancing our knowledge to specific compounds in different tissues can provide powerful opportunities, e.g. to study the physiology of migrating animals, which are hard to capture multiple times. The analysis of $A A$ isotope signatures of these animals from tissues with different incorporation rates can help to illuminate temporal variations and identify endogenous vs exogenous resources, without the need of multiple sampling points.

\section{Fractionation between tissues}

We were able to separate muscle and liver tissue by their carbon isotopic signature of AAs using ASCA (Figure 3). ASCA provided an alternative way to PCA and LDA as classical multivariate analysis and was especially useful in our case because we could incorporate the structure of our experiment into the model with time points and sample types as two separate factors, which is not possible in PCA ${ }^{46}$. This removed a lot of "noise" from the dietary isotope shift when investigating isotope fractionation between sample types and to identify AAs with high or low fractionation patterns. Highest loadings were observed for Gly and Ser with opposite trends, where Gly has the most negative loading and separates liver tissue from dietary and muscle samples. Ser and Ala have the most positive loading and separate muscle from both diet and liver. It also revealed low fractionation of Arg and Lys isotope signatures between all tissue types, as those two AAs have the lowest loading overall on both PCs. Based on the results from ASCA we decided to especially focus on these AAs during ANOVA between tissue types, since the fractionation should be independent from the observed isotope shift over time induced by dietary samples.

NEAAs can be synthesized de novo in higher organisms, leading to more fractionation during metabolism and nutrient flow from primary producers to top predators in a given food web ${ }^{29}$. Our study however shows low isotope fractionations in general for NEAAs between diets and fish tissue and supports the idea of isotope routing for NEAAs when animals are fed protein rich diets, which is energetically favorable compared to de novo synthesis. High isotope fractionations of above $5 \%$ of some NEAAs between diet and consumer, as described by other studies ${ }^{11,13}$, did not occur in our case, but smaller differences were 
observed. These differences mainly occurred between muscle and liver tissue and not directly between diet and muscle/liver. It is worth mentioning here that the isotopic differences between liver and muscle tissue did not directly result from metabolic differences leading to different fractionation patterns but were a consequence of liver tissue following the isotopic shift of dietary sources over time, whereas muscle tissue remains almost constant. The only cases where NEAAs in fish tissue were significantly different compared to dietary samples were for Ala, Gly and Ser. The fractionation of Ser was striking, with Ser in the liver being isotopically depleted not only compared to muscle but also compared to diets after 30 days. Catabolic pathways for Ser involve deamination to pyruvate, transamination with pyruvate to form hydroxy pyruvate and alanine and formation of Gly with tetrahydrofolate ${ }^{47}$. Additionally, participation of Gly into gluconeogenesis requires the conversion of Gly to Ser by serine hydroxymethyltransferase and both AAs participate further in sulfur and one-carbon metabolism ${ }^{16}$. These two AAs are also the only NEAAs in the liver showing abnormal isotopic behavior, with Ser being significantly depleted compared to muscle and diets, whereas Gly is the only AA in liver being enriched at later sampling days. The liver plays a major role in energy and amino acid metabolism by regulating and controlling both glycolysis and gluconeogenesis as well as synthesizing many of the NEAAs ${ }^{24}$. Synthesis of NEAA carbon backbones can occur from either glycolytic intermediates for Gly, Ser and Ala or from Krebs cycle intermediates for Asp and $\mathrm{Glu}^{48}$. It is, however, hard to estimate the contribution of isotopic fractionation for each of these pathways, not only because intrinsic fractionation and mass flow are unknown, but also because metabolic pathways are often intertwined and hard to separate in living organisms at natural abundance level. Enzymatic reactions and catabolism of nutrients are usually associated with discrimination against the heavier isotopes, in this case ${ }^{13} \mathrm{C}$, leading to depleted signatures in the product, while the educt of the reaction becomes isotopically ${ }^{13} \mathrm{C}$-enriched ${ }^{49}$. The small enrichment of Gly observed in liver could therefore be indicative of extensive conversion of Gly in this tissue to other compounds, with Ser being one candidate and resulting in a depletion of its isotope signature. This system could even be extended to include Thr as an EAA. Animals are able to catabolize substantial amounts of Thr to yield Gly by L-threonine 3-dehydrogenase ${ }^{50}$. Applying the same principle of isotope fractionation during enzymatic reactions, Thr could theoretically be enriched in consumer tissue compared to diets and although Thr in fish liver was not significantly different compared to fish diet, liver tissue showed tendencies to be isotopically enriched in its carbon isotope signature by 0.4 to $1.3 \%$. It is, however, a far stretch at this point to account the small difference in Thr and Gly to specific metabolic or enzymatic reactions, especially since metabolism of Gly and Ser involve several different pathways. Future research and controlled feeding experiments could focus on using isotopically labeled substitutes to track individual metabolic pathways, such as the catabolism of Thr to Gly and Ser. Thr could be especially useful in that regard, since it can`t be synthesized in higher organisms and has to be taken up by diets. Pro was the only NEAA showing no difference at all over the sampling period, which points more to a behavior like an EAA being rooted by diets. As mentioned in the introduction, Pro can be synthesized in higher organisms, but the classification as a NEAA is sometimes misleading as metabolic requirements might heavily outmatch de novo synthesis. Our results indicate that fish fed on high protein 
content incorporate Pro mostly from dietary sources leading to no or very little fractionation in both liver and muscle tissue.

Phe and Tyr were the only EAAs showing different isotope signatures between diet and fish tissue, which is surprising for Phe since it also needs to be directly taken up and rooted from dietary sources. Tyr however is synthesized from Phe and the depleted isotopic signature of Tyr compared to diets could therefore also be explained by enzymatic fractionation discriminating against the heavy carbon isotopes. Arg and Lys were the EAAs with lowest fractionation overall, strongly following the trend of dietary isotope signatures even in muscle tissue over short time periods. Arg is highly abundant in fish protein and tissue fluid ${ }^{16}$, serving as a precursor for the synthesis of proteins, nitric oxide, urea, Pro, Glu and creatine ${ }^{51}$. Arg is of special interest here because it is not often reported in the literature, since most studies apply GC-IRMS for measurement of carbon isotope signatures of AAs, which requires derivatization and results in the loss of Arg ${ }^{52,53}$. Because of the high abundance, demand and turnover of dietary Arg in fish leading to very little fractionation even in muscle tissue over a short-term dietary isotope shift, it could serve as another proxy in addition to Phe, Leu, lle and Lys ${ }^{10-13}$ to track carbon sources from primary producers in muscle or liver tissue. Identifying EAAs with very low fractionation patterns is pivotal for ecological studies using isotope fingerprinting to better constrain the source of endmember signatures and study the carbon flow in terrestrial and oceanic environments $3,8,9,13-15,29$. Although EAAs cannot be synthesized by eukaryotes, the fundamental assumption that these constituents must be solely sourced from dietary protein resulting in low isotope fractionation of $\sim 0 \%$ has not always been met. There are studies suggesting that gut microbes can contribute to the homeostasis of EAAs in animals by de novo synthesis, which of course complicates their use as stable and robust proxies for isotope fingerprinting 29,54-57. The contribution of microorganisms in the gut of sticklebacks to the EAAs homeostasis could explain the observed difference of Phe and Tyr isotope signatures in fish muscle and liver tissue compared to diets and highlights the importance to experimentally explore the variation of EAA isotope fractionation by gut microbiome. According to our results, Arg and Lys might therefore be better suited in sticklebacks and possibly other teleost species to estimate their carbon flow and allocate resource consumption, if the contribution of gut symbionts is of no interest.

\section{Isotope fractionation of Histidine}

His showed overall the highest fractionation among the analyzed AA. This represents an unusual outcome, as EAAs are taken up directly from diet and incorporated into consumer tissue with very low trophic discrimination. The previously mentioned de novo synthesis and contribution of EAAs from gut microbes could of course be one explanation for the highly ${ }^{13} \mathrm{C}$-enriched isotope signatures of His in in stickleback liver and muscle tissue. Other reasons could include the enzyme histidine decarboxylase, which is produced by bacteria and causes histamine fish poisoning ${ }^{58,59}$. It has been speculated before that histidine decarboxylase might still be released from autolyzing of bacterial cells during and after freeze drying and convert histidine to histamine ${ }^{60}$. Enzymatic reactions are known to cause isotopic 
fractionation, which usually discriminates against the heavier isotope ${ }^{49}$ and could result in enriched isotope signature of the remaining histidine in fish tissue. Interestingly, an early study investigating histamine concentrations during storage of flesh and liver tissue of mackerel under different conditions showed a higher increase of histamine concentrations in the liver ${ }^{61}$, which would fit to the higher isotope values of histidine in stickleback liver compared to muscle tissue, if it was caused by enzymatic reactions. Testing this, however, would require histamine isotope measurements in dietary and fish samples to compare isotope signatures and was out of scope of this study. Another explanation would be that sticklebacks fed on mosquito larvae lack dietary histidine to match their metabolic requirements. We did not directly measure the protein and AA content of dietary mosquito larvae, but His has been mentioned in the literature as a more frequent limiting $A A$ when animals are fed with insect meals ${ }^{62}$. His is an important amino acid for growth, tissue formation and hemoglobin synthesis in fish ${ }^{63}$ and a lack of dietary His may lead to increased fractionation in consumer tissue due to catabolism during starvation. There is however no consensus so far on the isotopic effect of starvation or nutritional stress on consumer tissue ${ }^{64}$ especially for single compounds, and such effects could be subject for further studies.

\section{Conclusion}

Studying the isotope signature of AAs between diet and consumer gained much attraction during the last years, yet our knowledge of the fundamental principles behind isotope incorporation and fractionation of individual substitutes is still limited. Our study shows that direct isotope routing even of NEAAs might still be the preferred way for nutrient assimilation when fish are fed with high protein diets, and it seems that there are not only differences in isotopic turnover rates between muscle and liver, but also that individual AAs in these tissues show different isotopic behaviors based on their metabolic role. However, investigating isotope fractionation of AAs on natural abundance levels might not be the best approach to study fundamental incorporation and turnover patterns, since a lot of information might be lost to natural variations and measurement uncertainty. This calls for controlled feeding experiments with heavily ${ }^{13} \mathrm{C}$ enriched diets or single constituents to accurately track metabolic pathways. One good example to investigate could be the mentioned Thr, Gly and Ser catabolism in animals, and has the potential to greatly improve our understanding of nutrient assimilation and conversion, which is needed and long overdue for the correct analysis and interpretation of field data. On the other hand, it is promising to see that even small differences and fluctuations of isotope values, which are more realistically encountered in nature, can be investigated using CSIA of different sample materials.

\section{Materials And Methods \\ Feeding experiment}

Three-spined Sticklebacks were laboratory-raised offspring and reared in twelve $14 \mathrm{~L}$ tanks (VewaTech, Germany) as part of a parasitic infection experiment. Water was recirculated and held at $18^{\circ} \mathrm{C}$ with a $15 \mathrm{~h}$ 
light and $9 \mathrm{~h}$ dark cycle. Stickleback offspring were produced by in vitro fertilization from individuals collected from a brook in North-West Germany $\left(52^{\circ} 17^{\prime} 33.11^{\prime \prime} \mathrm{N}, 7^{\circ} 36^{\prime} 46.48^{\prime \prime} \mathrm{E}\right)$, about eight months old at the beginning of the experiment and fed daily with washed red mosquito larvae (Chironomidae) over the course of four months. Each lab tank contained twelve individuals, which were divided into two groups. The first group consisted of 3 individuals per tank, which were exposed to an uninfected copepod as a sham-exposed control group, while the other 9 individuals per tank were exposed to an infected copepod as a parasite-exposed group. Three complete lab tanks with 36 individuals were sampled on each sampling date $(30,60,90$ and 120 days post exposure) and five out of the fifteen sham-exposed individuals per sampling date were randomly selected for CSIA. Sub samples of mosquito larvae were taken once a week, stored at $-20^{\circ} \mathrm{C}$ and pooled between 1-30, 31-60, 61-90 and 91-120 days for CSIA. Insect larvae are considered to be a protein rich diet for fish, with a crude protein content of up to $60 \%$ and an adequate amino acid profile, although His, Lys or Try can be lacking depending on the used insect species ${ }^{62,65,66}$. Sticklebacks were starved $72 \mathrm{~h}$ prior sampling, anesthetized with MS 222 (Sigma-Aldrich, USA) and killed by decapitation. Liver and muscle tissue were collected without skin and bones and stored at $-20^{\circ} \mathrm{C}$ until further use. Sticklebacks were maintained and treated in accordance with the local animal welfare authorities and the EU Directive 2010/63/EU for animal experiments. All animal experiments described were approved by the 'State Agency for Nature, Environment and Consumer Protection' (LANUV) of North Rhine Westphalia, which includes the evaluation by an ethics committee, under the project number 87 51.04.2010.A297.

\section{Sample preparation}

Hydrolysis of amino acids for LC-IRMS analysis has been described in the literature 38,39,67. Approximately $5 \mathrm{mg}$ of sample material were weighed into $5 \mathrm{~mL}$ PTFE vials (CEM GmbH, Kamp-Lintfort, Germany) and $2.5 \mathrm{~mL}$ (1:500 ratio of mass to volume) of $6 \mathrm{M}$ hydrochloric acid ( $>99 \%$, Alfa Aesar, Kandel, Germany) were added. The vials were closed and kept in a UT 5042 drying oven (Heraeus, Hanau, Germany) at $110^{\circ} \mathrm{C}$ for $24 \mathrm{~h}$. The hydrolysate was filtered ( $0.2 \mu \mathrm{m}$ PTFE filter), evaporated to dryness under vacuum at $40^{\circ} \mathrm{C}$, reconstituted in $1 \mathrm{~mL}$ distilled water and filtered again into small $1.5 \mathrm{~mL}$ HPLC vials. The vials were frozen at $-20^{\circ} \mathrm{C}$ until LC-IRMS analysis. Glu and Asp are converted to their respective acidic form during this treatment and measured together with glutamic and aspartic acid. Tryptophan and cysteine are lost during acid hydrolysis and the amount of methionine was too low to be measured accurately.

\section{LC-IRMS analysis of AAs}

For simplicity, we used a classification of only EAA and NEAA as follows: NEAA; Alanine (Ala), Asparagine (Asp), Glutamine (Glu), Glycine (Gly), Proline (Pro), Serine (Ser) and EAA; Arginine (Arg), Histidine (His), Lysine (Lys), Phenylalanine (Phe), Tyrosine (Tyr), Threonine (Thr), placing the conditionally essential AAs Glu and Pro into the NEAA category and Tyr into the EAA category because of its close connection to Phe.

The analysis of individual AAs was performed on a Dionex Ultimate 3000 HPLC Pump (Thermo Fisher Scientific, Bremen, Germany) coupled to an Isolink Interface and Delta V Advantage mass spectrometer 
(Thermo Fisher Scientific, Bremen, Germany). Separation was achieved for 13 AAs (Figure S1, Supporting Information) with a mixed mode cation exchange column (Primesep A, $2.1 \mathrm{~mm} \mathrm{ID,} 250 \mathrm{~mm} \mathrm{~L}, 5 \mu \mathrm{m}$ particle size) from SIELC, which was in accordance to other studies employing the same separation technique $38,39,67$. The exact program is described in Raghaven et al. (2010) and uses a gradient from mobile phase $\mathrm{A}(100 \%$ water, $\mathrm{pH}$ ) $)$ to mobile phase $\mathrm{B}(0.3 \mathrm{M}$ sulfuric acid, $\mathrm{pH} 1.5)$ and column temperature was held at $30^{\circ} \mathrm{C}$. To preserve the HPLC column, which is very sensitive to $\mathrm{pH}$ values of over 7 , we adjusted the method to start with water $\mathrm{pH} 4$ as mobile phase $\mathrm{A}$. Flow rate of the mobile phase was set to $260 \mu \mathrm{Lmin}^{-1}$ and oxidation agents $\left(1.5 \mathrm{M}\right.$ orthophosphoric acid and $100 \mathrm{gL}^{-1}$ disodium peroxodisulfate, Merck, Darmstadt, Germany) were pumped at $25 \mu \mathrm{Lmin}^{-1}$ each. This resulted in an oxygen background of approximately $12 \mathrm{~V}$ on the first cup of the IRMS, which is the recommended value by the manufacturer to guarantee sufficient oxidation conditions. The experimental units were replicates of pooled dietary samples $(n=3)$ and biological replicates of stickleback tissues (liver and muscle, $n=5$ ) for each of the four sampling days taken at 30,60, 90 and 120 days were hydrolyzed, and the small sample amount of obtained stickleback tissue did not allow for within-individual replicate analysis. Each hydrolyzed sample was injected in triplicate into the LC-IRMS system and outliers were determined by Grubbs Test on a confidence level of 0.95 and excluded from further analysis. We calculated mean values and SD from triplicate injections to estimate instrumental precision before referencing our data. Instrumental precision was estimated with an average SD of $0.47 \%$ o for triplicate injections of all AAs, tissues, and sampling days $(n=674)$. SDs of triplicate injections of all AAs from either dietary samples $(0.51 \%, n=156)$, liver samples $(0.50 \%, n=258)$ and muscle samples $(0.41 \%, n=260)$ where almost equal over the complete sampling period. The robustness of sample preparation and hydrolysis was assessed by conducting replicate analysis $(n=3)$ of dietary samples, since these were the only samples providing enough material for multiple replicates. The average SD of replicate analysis was $0.36 \%$ ond therefore in the same range as triplicate injections. Twelve in-house amino acid standards (Ala, Asp, Arg, Glu, Gly, His, Lys, Pro, Phe, Ser, Thr and Tyr) were purchased with a purity of $>98 \%$ (Alfa Aesar, Kandel, Germany) and measured against seven certified international AA reference materials (L-Alanine, LGlutamic acid, USGS 64, USGS 66, L-Phenylalanine, L-Proline and L-Valine), purchased from Arndt Schimmelmann, Department of Earth and Atmospheric Sciences at Indiana University (Bloomington, IN, USA), on an Isoprime100 Elemental Analyser (Elementar Analysensysteme $\mathrm{GmbH}$, Langenselbold, Germany). A mix of the in-house standards with a concentration of $100 \mathrm{mgL}^{-1}$ for each amino acid was regularly measured in between sample runs and used to directly assign final isotope values on the VPDB scale. This procedure ensures accurate long-term isotope data and follows the identical treatment procedure ${ }^{68}$. Chromatograms were individually checked for proper background and peak detection. The automated dynamic background detection algorithm from ISODAT 2.0 software with a block width of 1000 (Lynch et al. 2011) was in many cases able to accurately estimate the background signal, but manual adjustments had to be made e.g., for closely eluding AAs or interference of matrix components.

\section{Data analysis}

Data analysis was done using Excel from Microsoft Office 365 ProPlus (Microsoft, Redmond, Washington, USA), Origin 2019 version 9.60 (OriginLab, Northampton, Massachusetts, USA) and Matlab 
R2021a (MathWorks Inc., Natick, Massachusetts, USA) with the PLS_Toolbox suite (Eigenvector Research Inc., Manson, WA). Isotope data are reported as mean $\delta^{13} \mathrm{C}_{\mathrm{AA}}$ values on the VPDB scale in permil (\%o) with its corresponding standard deviation (SD). Data was tested for normality with Kolmonogorov Smirnov-Test because of the small sample size on a confidence level of 0.95 , which was not violated for any given AA and sample. One-way analysis of variance (ANOVA) was used to test for differences of AAs over time points (days) and between tissue type. Using time points as an independent variable in ANOVA can be problematic because it is not strictly categorial, but each of our sample represents an independent fish individual which couldn't be sampled multiple times and is therefore not a repeated measure. The significance level a was set to 0.01 to compensate for the low number of biological replicates $(n=5$ for fish tissue) that were analyzed, and each ANOVA analysis was accompanied by Brown-Forsythe tests ( $\mathrm{a}$ $=0.05$ ) to check for equality of group variances and followed up with Tukey post hoc tests to identify significant differences between group values. Reducing the significance level avoids false-positive results for small sample sizes, but it consequently increases false-negative results, and we are therefore only discussing the most significant differences of our data, while smaller differences might be lost. We further conducted ANOVA simultaneous component analysis (ASCA) as a multivariate analysis in a design of experiment approach, with days and tissue as fixed factors and $\delta^{13} \mathrm{C}$ of each $\mathrm{AA}$ as multivariable. ASCA combines the principles of ANOVA and PCA ${ }^{46}$ and allows to investigate differences in the isotope signature between tissues without the influence of the expected isotope shift over time dominating our analysis. Isotope data were used without any transformation.

\section{Declarations}

\section{Data availability}

The datasets generated during and/or analysed during the current study are available in the Figshare repository: https://doi.org/10.6084/m9.figshare.17014220.v1

\section{Acknowledgements}

We thank the Deutsche Forschungsgemeinschaft (DFG, German Research Foundation) for funding this project SFB 1439/1 2021426547801.

\section{Author Contributions}

All authors contributed substantially to this study. The feeding experiment was designed and conducted by F.F., M.N., J.P.S. and J.K.. Isotope analysis was carried out by T.H. and S.K.. Data analysis and evaluation was done by T.H. with helpful input and support from S.K., M.N., M.A.J and T.C.S.. The manuscript was drafted by T.H. with substantial feedback and help from all other authors. 


\section{Competing interest}

The authors declare no competing interests.

\section{Additional information}

The present study was carried out in compliance with the ARRIVE guidelines (https://arriveguidelines.org/)

\section{References}

1. Newsome, S. D., Clementz, M. T. \& Koch, P. L. Using stable isotope biogeochemistry to study marine mammal ecology. Marine Mammal Science 26, 509-572, doi:10.1111/j.1748-7692.2009.00354.x (2010).

2. Layman, C. A. et al. Applying stable isotopes to examine food-web structure: an overview of analytical tools. Biol Rev Camb Philos Soc 87, 545-562, doi:10.1111/j.1469-185X.2011.00208.x (2011).

3. Larsen, T. et al. Tracing carbon sources through aquatic and terrestrial food webs using amino acid stable isotope fingerprinting. PLoS One 8, e73441, doi:10.1371/journal.pone.0073441 (2013).

4. Post, D. M. Using stable isotopes to estimate trophic position: models, methods and assumptions. Ecology 83, 703-718 (2002).

5. Inger, R. \& Bearhop, S. Applications of stable isotope analyses to avian ecology. Ibis $150,447-461$ (2008).

6. McCutchan, J. H., Lewis, W. M., Kendall, C. \& McGrath, C. C. Variation in trophic shift for stable isotope ratios of carbon, nitrogen, and sulfur. OIKOS 102, 378-390 (2003).

7. Olive, P. J. W., Pinnegar, J. K., Polunin, N. V. C., Richards, G. \& Welch, R. Isotope trophic-step fractionation: a dynamic equilibrium model. Journal of Animal Ecology 72, 608-617 (2003).

8. McMahon, K. W., Polito, M. J., Abel, S., McCarthy, M. D. \& Thorrold, S. R. Carbon and nitrogen isotope fractionation of amino acids in an avian marine predator, the gentoo penguin (Pygoscelis papua). Ecol Evo/ 5, 1278-1290, doi:10.1002/ece3.1437 (2015).

9. Webb, E. C. et al. Compound-specific amino acid isotopic proxies for distinguishing between terrestrial and aquatic resource consumption. Archaeological and Anthropological Sciences 10, 118, doi:10.1007/s12520-015-0309-5 (2016).

10. Whiteman, J. P., Kim, S. L., McMahon, K. W., Koch, P. L. \& Newsome, S. D. Amino acid isotope discrimination factors for a carnivore: physiological insights from leopard sharks and their diet. Oecologia 188, 977-989, doi:10.1007/s00442-018-4276-2 (2018).

11. Rogers, M., Bare, R., Gray, A., Scott-Moelder, T. \& Heintz, R. Assessment of two feeds on survival, proximate composition, and amino acid carbon isotope discrimination in hatchery-reared Chinook 
salmon. Fisheries Research 219, doi:10.1016/j.fishres.2019.06.001 (2019).

12. Wang, Y. V., Wan, A. H. L., Krogdahl, A., Johnson, M. \& Larsen, T. (13)C values of glycolytic amino acids as indicators of carbohydrate utilization in carnivorous fish. PeerJ 7, e7701, doi:10.7717/peerj.7701 (2019).

13. McMahon, K. W., Fogel, M. L., Elsdon, T. S. \& Thorrold, S. R. Carbon isotope fractionation of amino acids in fish muscle reflects biosynthesis and isotopic routing from dietary protein. J Anim Ecol 79, 1132-1141, doi:10.1111/j.1365-2656.2010.01722.x (2010).

14. McMahon, K. W., Thorrold, S. R., Houghton, L. A. \& Berumen, M. L. Tracing carbon flow through coral reef food webs using a compound-specific stable isotope approach. Oecologia 180, 809-821, doi:10.1007/s00442-015-3475-3 (2016).

15. Wang, Y. V. et al. Know your fish: A novel compound-specific isotope approach for tracing wild and farmed salmon. Food Chem 256, 380-389, doi:10.1016/j.foodchem.2018.02.095 (2018).

16. Li, P., Mai, K., Trushenski, J. \& Wu, G. New developments in fish amino acid nutrition: towards functional and environmentally oriented aquafeeds. Amino Acids 37, 43-53, doi:10.1007/s00726008-0171-1 (2009).

17. Boecklen, W. J., Yarnes, C. T., Cook, B. A. \& James, A. C. On the Use of Stable Isotopes in Trophic Ecology. Annu. Rev. Ecol. Evol. Syst. 42, 411-440, doi:10.1146/annurev-ecolsys-102209-144726 (2011).

18. Logan, J. M. \& Lutcavage, M. E. Stable isotope dynamics in elasmobranch fishes. Hydrobiologia 644, 231-244, doi:10.1007/s10750-010-0120-3 (2010).

19. Madigan, D. J. et al. Tissue turnover rates and isotopic trophic discrimination factors in the endothermic teleost, pacific bluefin tuna (Thunnus orientalis). PLoS One 7, e49220, doi:10.1371/journal.pone.0049220 (2012).

20. Perga, M. E. \& Gerdeaux, D. 'Are fish what they eat' all year round? Oecologia 144, 598-606, doi:10.1007/s00442-005-0069-5 (2005).

21. Skinner, M. M., Cross, B. K. \& Moore, B. C. Estimating in situ isotopic turnover in Rainbow Trout (Oncorhynchus mykiss) muscle and liver tissue. Journal of Freshwater Ecology 32, 209-217, doi:10.1080/02705060.2016.1259127 (2016).

22. Sponheimer, M. et al. Turnover of stable carbon isotopes in the muscle, liver, and breath $\mathrm{CO} 2$ of alpacas (Lama pacos). Rapid Commun Mass Spectrom 20, 1395-1399, doi:10.1002/rcm.2454 (2006).

23. Kaushik, S. J. \& Seiliez, I. Protein and amino acid nutrition and metabolism in fish: current knowledge and future needs. Aquaculture Research 41, 322-332, doi:10.1111/j.1365-2109.2009.02174.x (2010).

24. Hou, Y., Hu, S., Li, X., He, W. \& Wu, G. Amino Acid Metabolism in the Liver: Nutritional and Physiological Significance. Vol. 1265 (2020).

25. Rui, L. Energy metabolism in the liver. Compr Physio/ 4, 177-197, doi:10.1002/cphy.c130024 (2014). 
26. Schutz, Y. Protein Turnover, Ureagenesis and Gluconeogenesis. Int. J. Vitam. Nutr. Res. 81, 101-107, doi:10.1024/0300 (2011).

27. Marks, V. in Encyclopedia of Human Nutrition 387-392 (2013).

28. Jim, S., Jones, V., Ambrose, S. H. \& Evershed, R. P. Quantifying dietary macronutrient sources of carbon for bone collagen biosynthesis using natural abundance stable carbon isotope analysis. $\mathrm{Br} \mathrm{J}$ Nutr 95, 1055-1062, doi:10.1079/bjn20051685 (2006).

29. Newsome, S. D., Fogel, M. L., Kelly, L. \& del Rio, C. M. Contributions of direct incorporation from diet and microbial amino acids to protein synthesis in Nile tilapia. Functional Ecology 25, 1051-1062, doi:10.1111/j.1365-2435.2011.01866.x (2011).

30. Gannes, L. Z., O'Brien, D. M. \& Del Rio, C. M. Stable isotopes in animal ecology: assumptions, caveats and a call for more laboratory experiments. Ecology 78, 1271-1276 (1997).

31. Martinez del Rio, C. M., Wolf, N., Carleton, S. A. \& Gannes, L. Z. Isotopic ecology ten years after a call for more laboratory experiments. Biol Rev Camb Philos Soc 84, 91-111, doi:10.1111/j.1469185X.2008.00064.x (2009).

32. Hendry, A. P., Peichel, C. L., Boughman, J. W., Matthews, B. \& Nosil, P. Stickleback research: the now and the next. EVOL ECOL RES 15, 111-141 (2013).

33. Fang, B., Merila, J., Ribeiro, F., Alexandre, C. M. \& Momigliano, P. Worldwide phylogeny of three-spined sticklebacks. Mol Phylogenet Evol 127, 613-625, doi:10.1016/j.ympev.2018.06.008 (2018).

34. Kume, M. \& Kitano, J. Genetic and stable isotope analyses of threespine stickleback from the Bering and Chukchi seas. Ichthyological Research 64, 478-480, doi:10.1007/s10228-017-0580-9 (2017).

35. Reimchen, T. E., Ingram, T. \& Hansen, S. C. Assessing niche differences of sex, armour and asymmetry phenotypes using stable isotope analyses in Haida Gwaii sticklebacks. Behaviour 145, 561-577 (2008).

36. Pinnegar, J. Unusual stable isotope fractionation patterns observed for fish host-parasite trophic relationships. Journal of Fish Biology 59, 494-503, doi:10.1006/jfbi.2001.1660 (2001).

37. Power, M. \& Klein, G. M. Fish host-cestode parasite stable isotope enrichment patterns in marine, estuarine and freshwater fishes from northern Canada. Isotopes Environ. Health Stud. 40, 257-266 (2004).

38. McCullagh, J. S., Juchelka, D. \& Hedges, R. E. Analysis of amino acid 13C abundance from human and faunal bone collagen using liquid chromatography/isotope ratio mass spectrometry. Rapid Commun Mass Spectrom 20, 2761-2768, doi:10.1002/rcm.2651 (2006).

39. Raghavan, M., McCullagh, J. S., Lynnerup, N. \& Hedges, R. E. Amino acid delta13C analysis of hair proteins and bone collagen using liquid chromatography/isotope ratio mass spectrometry: paleodietary implications from intra-individual comparisons. Rapid Commun Mass Spectrom 24, 541-548, doi:10.1002/rcm.4398 (2010).

40. Li, X., Zheng, S. \& Wu, G. Nutrition and metabolism of glutamate and glutamine in fish. Amino Acids 52, 671-691, doi:10.1007/s00726-020-02851-2 (2020). 
41. Newsome, S. D., del Rio, C. M., Bearhop, S. \& Phillips, D. L. A niche for isotopic ecology. Front Ecol Environ 5, 429-436, doi:10.1890/060150.01 (2007).

42. Voigt, C. C., Rex, K., Michener, R. H. \& Speakman, J. R. Nutrient routing in omnivorous animals tracked by stable carbon isotopes in tissue and exhaled breath. Oecologia 157, 31-40, doi:10.1007/s00442008-1057-3 (2008).

43. Tieszen, L. L., Boutton, T. W., Tesdahl, K. G. \& Slade, N. A. Fractionation and turnover of stable carbon isotopes in animal tissues: Implications for $613 \mathrm{C}$ analysis of diet. Oecologia 57, 21-37 (1983).

44. Cerling, T. E. et al. Determining biological tissue turnover using stable isotopes: the reaction progress variable. Oecologia 151, 175-189, doi:10.1007/s00442-006-0571-4 (2007).

45. Martínez del Rio, C. \& Carleton, S. A. How fast and how faithful: the dynamics of isotopic incorporation into animal tissues: Fig. 1. Journal of Mammalogy 93, 353-359, doi:10.1644/11mamm-s-165.1 (2012).

46. Bertinetto, C., Engel, J. \& Jansen, J. ANOVA simultaneous component analysis: A tutorial review. Anal Chim Acta X 6, 100061, doi:10.1016/j.acax.2020.100061 (2020).

47. Walton, M. J. \& Cowey, C. B. Aspects of intermediary metabolism in salmonid fish. Comp. Biochem. Physiol 73B, 59-79 (1982).

48. Fernandes, R., Nadeau, M.-J. \& Grootes, P. M. Macronutrient-based model for dietary carbon routing in bone collagen and bioapatite. Archaeol Anthropol Sci 4, 291-301, doi:10.1007/s12520-012-0102-7 (2012).

49. Ohkouchi, N., Ogawa, N. O., Chikaraishi, Y., Tanaka, H. \& Wada, E. Biochemical and physiological bases for the use of carbon and nitrogen isotopes in environmental and ecological studies. Progress in Earth and Planetary Science 2, 1-17, doi:10.1186/s40645-015-0032-y (2015).

50. House, J. D., Hall, B. N. \& Brosnan, J. T. Threonine metabolism in isolated rat hepatocytes. Am J Physiol Endocrinol Metab 281, E1300-E1307 (2001).

51. Wu, G. \& Morris, M. Arginine metabolism: nitric oxide and beyond. Biochem. J. 336, 1-17 (1998).

52. Metges, C. C., Petzke, K. J. \& Henning, U. Gas Chromatography/Combustion/Isotope Ratio Mass Spectrometric Comparison of N-Acetyl- and N-Pivaloyl Amino Acid Esters to Measure 15N Isotopic Abundances in Physiological Samples: A Pilot Study on Amino Acid Synthesis in the Upper Gastrointestinal Tract of Minipigs. J. Mass Spectrom. 31, 367-376 (1996).

53. Dunn, P. J., Honch, N. V. \& Evershed, R. P. Comparison of liquid chromatography-isotope ratio mass spectrometry (LC/IRMS) and gas chromatography-combustion-isotope ratio mass spectrometry (GC/C/IRMS) for the determination of collagen amino acid delta13C values for palaeodietary and palaeoecological reconstruction. Rapid Commun Mass Spectrom 25, 2995-3011, doi:10.1002/rcm.5174 (2011).

54. Ayayee, P. A., Jones, S. C. \& Sabree, Z. L. Can (13)C stable isotope analysis uncover essential amino acid provisioning by termite-associated gut microbes? PeerJ 3, e1218, doi:10.7717/peerj.1218 (2015). 
55. Ayayee, P. A., Larsen, T. \& Sabree, Z. Symbiotic essential amino acids provisioning in the American cockroach, Periplaneta americana (Linnaeus) under various dietary conditions. PeerJ 4, e2046, doi:10.7717/peerj.2046 (2016).

56. Larsen, T. et al. The dominant detritus-feeding invertebrate in Arctic peat soils derives its essential amino acids from gut symbionts. J Anim Eco/ 85, 1275-1285, doi:10.1111/1365-2656.12563 (2016).

57. Romero-Romero, S., Miller, E. C., Black, J. A., Popp, B. N. \& Drazen, J. C. Abyssal deposit feeders are secondary consumers of detritus and rely on nutrition derived from microbial communities in their guts. Sci Rep 11, 12594, doi:10.1038/s41598-021-91927-4 (2021).

58. Tsai, Y. et al. Histamine contents of fermented fish products in Taiwan and isolation of histamineforming bacteria. Food Chemistry 98, 64-70, doi:10.1016/j.foodchem.2005.04.036 (2006).

59. Landete, J. M., De las Rivas, B., Marcobal, A. \& Munoz, R. Updated molecular knowledge about histamine biosynthesis by bacteria. Crit Rev Food Sci Nutr 48, 697-714, doi:10.1080/10408390701639041 (2008).

60. Kanki, M., Yoda, T., Tsukamoto, T. \& Baba, E. Histidine decarboxylases and their role in accumulation of histamine in tuna and dried saury. Appl Environ Microbiol 73, 1467-1473, doi:10.1128/AEM.01907-06 (2007).

61. Fernandez-Salguero, J. \& Mackie, I. M. Histidine metabolism in mackerel (Scomber scombrus). Studies on histidine decarboxylase activity and histamine formation during storage of flesh and liver under sterile and non-sterile conditions. J. Fd Technol. 14, 131-139 (1979).

62. Sánchez-Muros, M.-J., Barroso, F. G. \& Manzano-Agugliaro, F. Insect meal as renewable source of food for animal feeding: a review. Journal of Cleaner Production 65, 16-27, doi:10.1016/j.jclepro.2013.11.068 (2014).

63. Khan, M. A. Histidine Requirement of Cultivable Fish Species: A Review. Oceanogr Fish Open Access J 8, 1-7, doi:10.19080/ofoaj.2018.08.555746 (2018).

64. Hatch, K. A. in Comparative Physiology of Fasting, Starvation, and Food Limitation Ch. Chapter 20, 337-364 (2012).

65. Nogales-Mérida, S. et al. Insect meals in fish nutrition. Reviews in Aquaculture 11, 1080-1103, doi:10.1111/raq.12281 (2018).

66. Thongprajukaew, K., Pettawee, S., Muangthong, S., Saekhow, S. \& Phromkunthong, W. Freeze-dried forms of mosquito larvae for feeding of Siamese fighting fish (Betta splendensRegan, 1910). Aquaculture Research 50, 296-303, doi:10.1111/are.13897 (2018).

67. Jackson, G. P., An, Y., Konstantynova, K. I. \& Rashaid, A. H. Biometrics from the carbon isotope ratio analysis of amino acids in human hair. Sci Justice 55, 43-50, doi:10.1016/j.scijus.2014.07.002 (2015).

68. Werner, R. A. \& Brand, W. A. Referencing strategies and techniques in stable isotope ratio analysis. Rapid Commun Mass Spectrom 15, 501-519, doi:10.1002/rcm.258 (2001). 


\section{Figures}

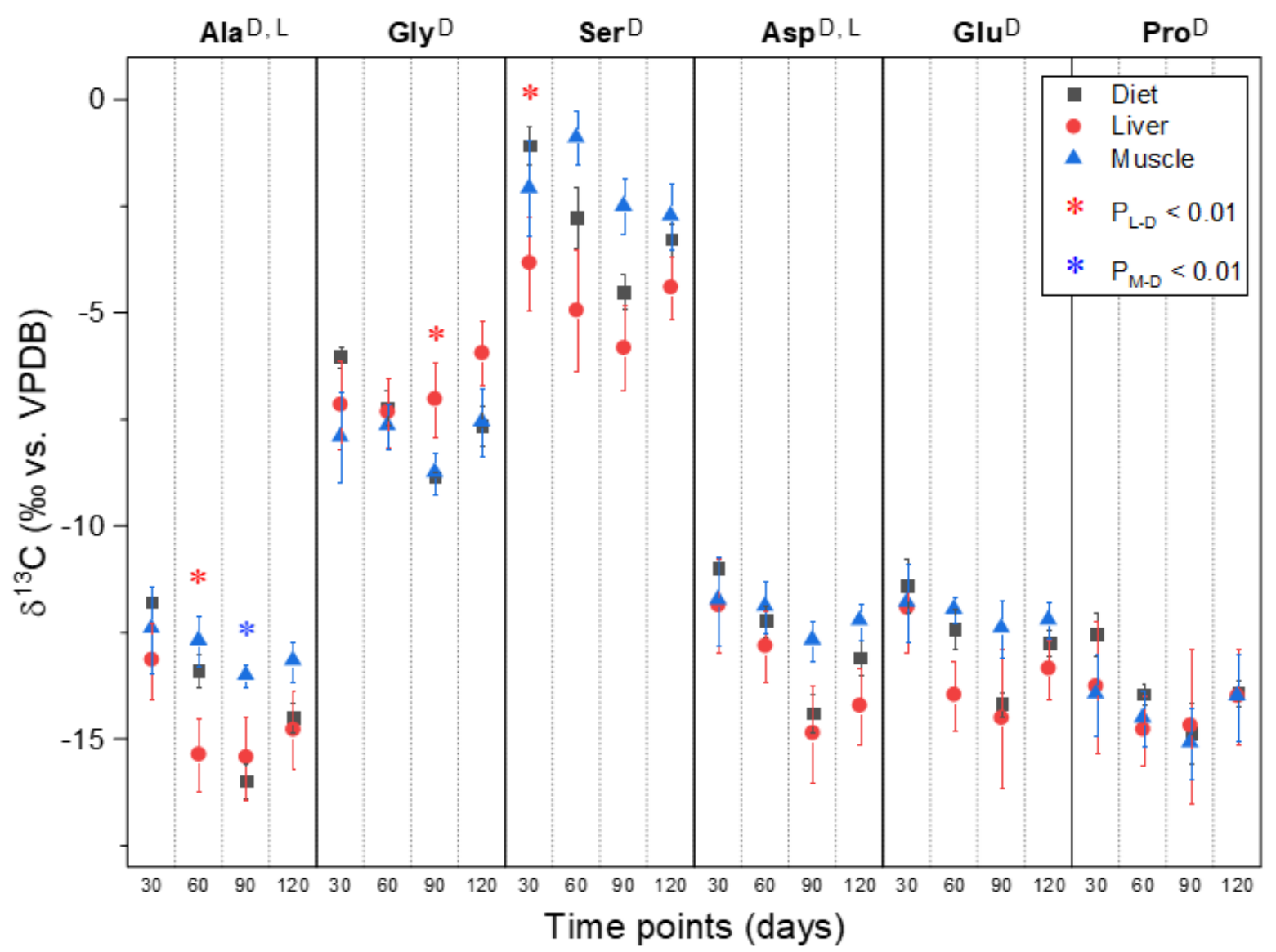

Figure 1: Carbon isotope signatures of NEAAs. Data is given as average $\delta^{13} \mathrm{C} \pm$ $\mathrm{SD}(\mathrm{n}=5)$ in \%o over the course of the feeding experiment for diet ( $\square)$, muscle $(\boldsymbol{\Delta})$ and liver (๑). Superscript letters next to AA abbreviations indicate significant differences between time points (days) of each sample type $(D=$ Diet, $M=$ Muscle, $L=$ Liver; oneway ANOVA, $p<0.01$, Table $S 2$, Supporting Information), whereas colored asterisks over sample data indicate significant differences between Liver-Diet (*) and MuscleDiet (*) on the respective time point (one-way ANOVA, $p<0.01$, Table S3, Supporting Information). Ala and Asp in the liver were the only NEAA showing a significant isotope shift over time, similar to dietary samples. Mean differences between liver and diet were significant for Ser after 30 days, Gly after 90 days and Ala after 60 days. Mean differences between muscle and diet were only significant for Ala after 90 days. Asp, Glu and Pro did not show significant fractionations between fish and diet.

Figure 1

Please See image above for figure legend. 


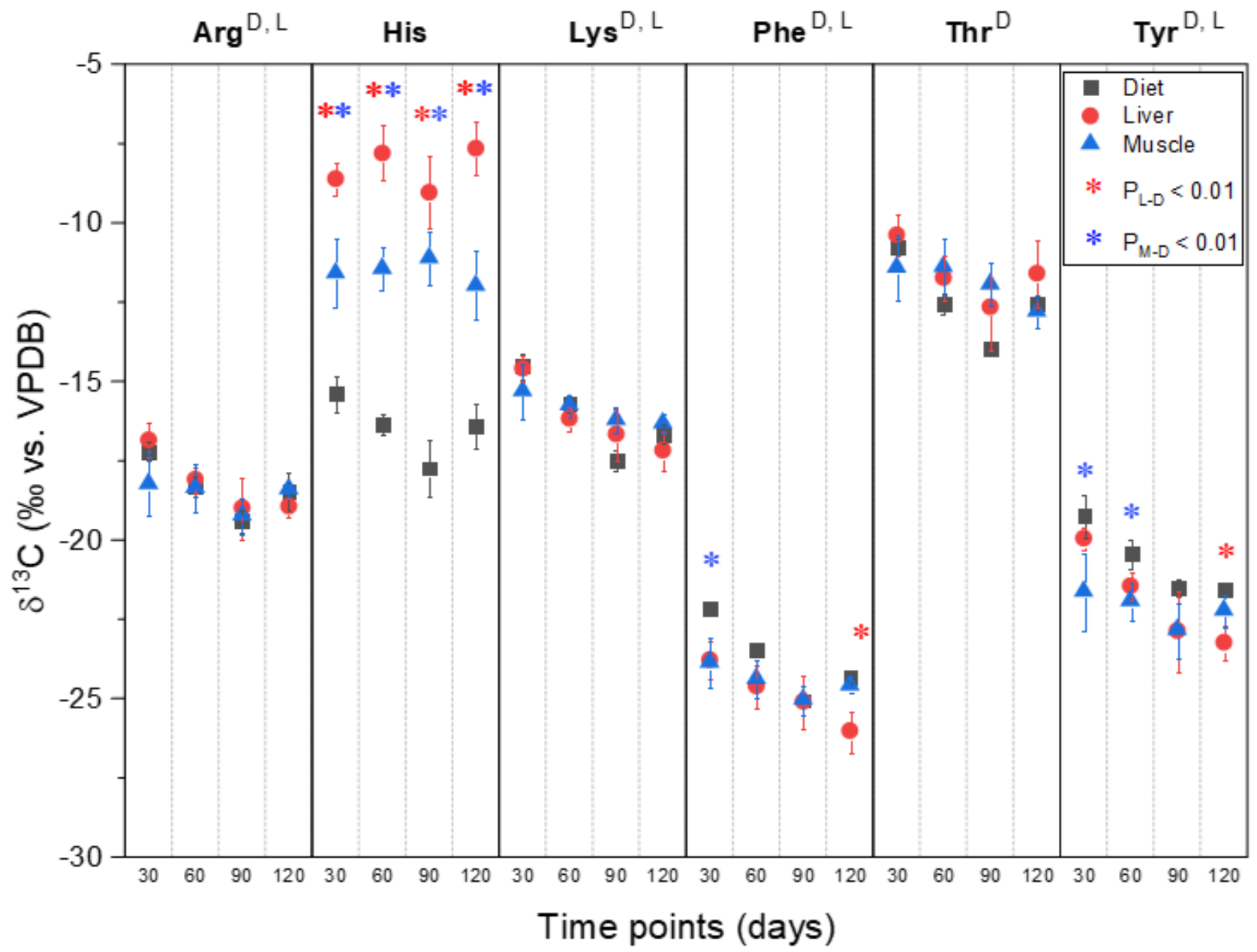

Figure 2: Carbon isotope signatures of EAAs. Data is given as average $\delta^{13} \mathrm{C} \pm \mathrm{SD}$ $(n=5)$ in \%o over the course of the feeding experiment. Symbols and indices are as in Figure 1. All dietary AAs except His and all liver AAs except His and Thr show significant differences over the sampling period (one-way ANOVA, $p<0.01$, Table S2, Supporting Information). His shows the overall highest fractionation of all AAs, which is very unusual for an EAA and will be discussed separately. Phe and Tyr show tendencies to be isotopically ${ }^{13} \mathrm{C}$-depleted between fish tissue and diets (one-way ANOVA, $p<0.01$, Table 3, Supporting Information). Arg, Lys and Thr show no isotopic differences between tissue types.

Figure 2

Please See image above for figure legend. 


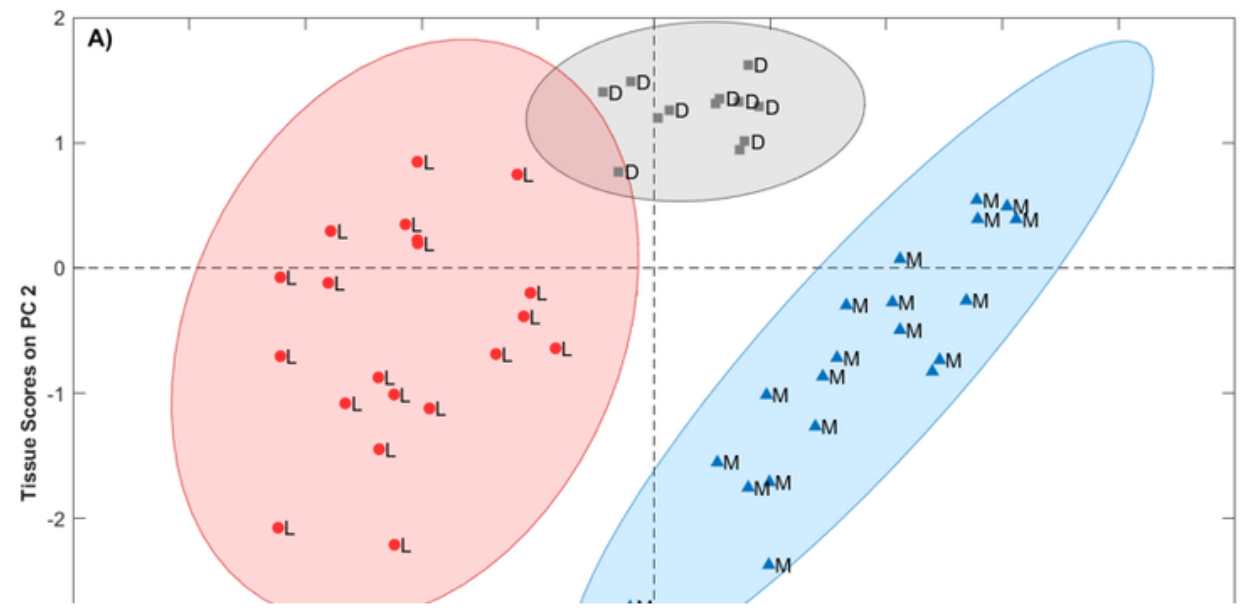

Figure 3

\section{Supplementary Files}

This is a list of supplementary files associated with this preprint. Click to download.

- SupportingInformation.docx 